\title{
INFLUÊNCIA DA CATÁLISE ÁCIDA E BÁSICA NA PREPARAÇÃO DA SÍLICA FUNCIONALIZADA PELO MÉTODO SOL-GEL
}

Eduardo José Nassar*

Universidade de Franca, CP 82, 14404-600 Franca - SP

Younès Messaddeq e Sidney José Lima Ribeiro

Instituto de Química, Universidade Estadual Paulista, CP 355, 14801-970 Araraquara - SP

Recebido em 29/9/00; aceito em 6/7/01

\begin{abstract}
BASE AND ACID CATALYSIS IN THE PREPARATION OF FUNCTIONALIZED SILICA OBTAIN BY SOL-GEL METHOD. Some aspects of the chemistry involved in the preparation and characterization of functionalized silicon oxide by sol-gel method are considered in this work. The synthesis was performed with different silicon alcoxide precursors and the influence of the acid and basic catalyst was investigated. Characterization was performed by infrared absorption spectroscopy, elemental analysis and ${ }^{29} \mathrm{Si}$ NMR. Infrared data show Si-C and $-\mathrm{CH}_{2}-$ vibrational modes at 1250 to 1280 and 2920 to $2940 \mathrm{~cm}^{-1}$, respectively. The elemental analysis confirmed the presence of organic groups in the inorganic silica network. ${ }^{29} \mathrm{Si} \mathrm{NMR}$ results show different hydrolisys depending on the acid or base catalysis.
\end{abstract}

Keywords: sol-gel; $\mathrm{RMN}{ }^{29} \mathrm{Si}$ and ${ }^{13} \mathrm{C}$; silica.

\section{INTRODUÇÃO}

A sílica é um polímero inorgânico, consistindo de grupos siloxanos ( $\mathrm{Si}-\mathrm{O}-\mathrm{Si})$ em seu interior, e de grupos silanóis ( $\mathrm{Si}-\mathrm{OH})$ em sua superfície ${ }^{1}$. A sílica forma uma das classes de substâncias inorgânicas mais utilizadas como suporte de uma variedade de sistemas com diferentes ramos de aplicação. A principal propriedade estudada na sílica está relacionada à sua superfície, a qual possui um considerável interesse quanto aos estudos da suas propriedades de adsorsão de moléculas ou íons ${ }^{2-7}$.

A sílica amorfa pode ser preparada pelo método sol-gel, a partir de precursores alcóxidos. A possibilidade de modificação da superfície do materials proporciona uma variedade muito grande de propriedades abrangendo um campo vasto de aplicações como em catálise, cromatografia e preparação de materiais luminescentes .Essa modificação da superfície pode ser feita através da condensação de alcoxissilano e/ou na preparação da sílica ${ }^{8-12}$.

Desta forma a sílica tem sido utilizada para imobilização de íons e compostos de terras raras ${ }^{13-15}$ com o intuito de estudar o aumento no rendimento quântico desses íons para possíveis aplicações como "fósforos". Recentemente, a sílica preparada pelo método sol-gel vem sendo estudada para aplicações como "fósforos" sem a presença de ativadores metálicos ${ }^{17}$. A grande vantagem na utilização destes "fósforos" reside na ausência de vapores de mercúrio existente nas lâmpadas fluorescentes atuais. Isto poderá conduzir a uma ampla variedade de materiais isento da toxidez dos metais.

Neste trabalho foram sintetizadas sílicas a partir do processo solgel com diferentes tipos de alcóxidos, utilizando a catálise ácida e básica. As sílicas tiveram como base o tetraetilortossilicato (TEOS). Ao TEOS foram adicionados, separadamente, metiltrimetoxissilano (MTMS), aminopropiltrietoxissilano (APTS), cloropropiltrimetoxissilano (TMOSCl) e água.

\footnotetext{
* e-mail: ejnassar@unifran.br
}

\section{PARTE EXPERIMENTAL}

\section{Preparação das sílicas}

As sílicas foram preparadas baseado no método descrito na literatura?.

A Tabela 1 apresenta as composições em mols das amostras de sílica preparadas com diferentes alcóxidos de silício.

Tabela 1. Composição inicial em mols dos reagentes.

\begin{tabular}{|c|c|c|c|c|c|c|c|}
\hline amostra & EOS & MTMS & APTS & TMOSCl & $\mathrm{HCl}^{*}$ & $\mathrm{NH}_{4} \mathrm{OH}^{*}$ & $\mathrm{H}_{2} \mathrm{O}$ \\
\hline S1 & 1 & - & - & - & 1 & - & 10 \\
\hline S2 & 1 & - & - & - & - & 1 & 10 \\
\hline S3 & 1 & 1 & - & - & 1 & - & 10 \\
\hline S4 & 1 & 1 & - & - & - & 1 & 10 \\
\hline S5 & 1 & - & 1 & - & 1 & - & 10 \\
\hline S6 & 1 & - & 1 & - & - & 1 & 10 \\
\hline S7 & 1 & - & - & 1 & 1 & - & 10 \\
\hline S8 & 1 & - & - & 1 & - & 1 & 10 \\
\hline
\end{tabular}

* concentração em $10^{-3} \mathrm{mols} / \mathrm{dm}^{3}$.

As sílicas foram preparadas através do processo sol-gel. Ao TEOS foram adicionados os reagentes na seguinte ordem; alcóxidos (MTMS ou APTS ou TMOSCl), o catalisador; $\left(\mathrm{HCl}\right.$ ou $\left.\mathrm{NH}_{4} \mathrm{OH}\right)$ e por fim $\mathrm{H}_{2} \mathrm{O}$, utilizando etanol absoluto como solvente. Este procedimento foi realizado sob vigorosa agitação magnética por $30 \mathrm{~min}$. Os sóis foram deixados em repouso até formação dos sólidos, os quais foram triturados e secos à $50^{\circ} \mathrm{C}$ por $48 \mathrm{~h}$.

A Figura 1 apresenta um fluxograma experimental da preparação das amostras.

\section{Caracterização}

As amostras foram caracterizadas através dos espectros de absorção na região do infravermelho, realizados em um aparelho PerkinElmer, de 4000 à $500 \mathrm{~cm}^{-1} \mathrm{em}$ pastilhas de $\mathrm{KBr}$. 


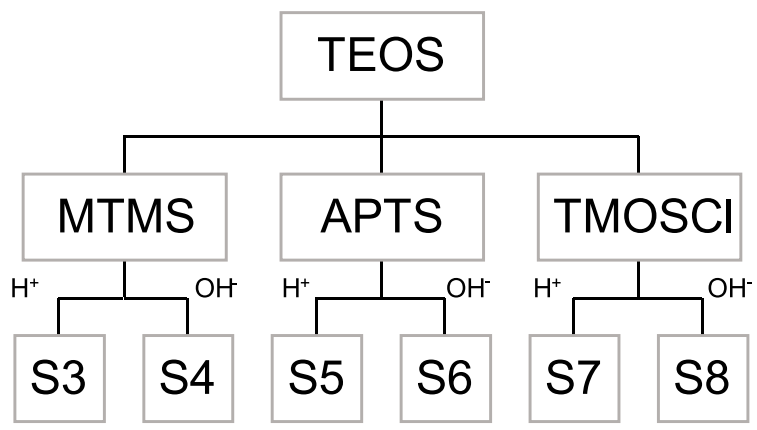

Figura 1. Fluxograma experimental

As análises elementares de $\mathrm{CNH}$ foram realizadas em um aparelho EA 1110 CHNSO-CE Instruments 0707.

A microscopia eletrônica de varredura (MEV) foi realizada em um microscópio eletrônico “ Jeol JSM-T330 A Microscópio de Varredura”.

Os espectros de ressonância magnética do ${ }^{29} \mathrm{Si}(59.5 \mathrm{MHz})$ e ${ }^{13} \mathrm{C}$ (75.4 MHz) foram obtidos em um espectrômetro INOVA 300 - Varian com rotor de nitreto de silício. O experimento ${ }^{29} \mathrm{Si}$ foi realizado usando um tempo de aquisição de $0,050 \mathrm{~s}$, pulso de $84,4^{\circ}$, com 156 repetições, uma razão de rotação de 2300 e um tempo médio de $2 \mathrm{~h} . \mathrm{O}^{13} \mathrm{C}$ foi realizado usando um tempo de aquisição de $0,050 \mathrm{~s}$, pulso de $28,4^{\circ}$, com 13300 repetições, uma razão de rotação de 6000 e um tempo médio de $15 \mathrm{~h}$.

\section{RESULTADOS E DISCUSSÃO}

Na Figura 2 são apresentadas fórmulas estruturais do alcóxidos utilizados.<smiles>CCO[Si](OCC)(OCC)OCC</smiles>

TEOS<smiles>CC[Si](OC)(OC)OC</smiles>

TMOSCl<smiles>CO[Si](C)(OC)OC</smiles>

MTMS<smiles>CCO[Si](CN)(OCC)OCC</smiles>

APTS
Figura 2. Fórmulas estruturais dos alcóxidos de silício

Os resultados calculado e experimental da análise elementar estão apresentados na Tabela 2.

S1 e S2: Essas amostras foram preparadas a partir do TEOS, a análise elementar não indicou a presença de $\mathrm{C}, \mathrm{H}$ ou $\mathrm{N}$. Isto já era esperado devido ao fato que a hidrólise do TEOS dá origem apenas ao polímero inorgânico. O subproduto da hidrólise é o etanol, o qual é eliminado durante o processo de secagem da sílica.

S3: A porcentagem de $\mathrm{C}$ e $\mathrm{H}$ é coerente com a razão molar 1:1 dos reagentes de partida. $\mathrm{Na}$ amostras $\mathbf{S 4}$ a porcentagem de $\mathrm{C}$ é menor que o esperado, isto é um indicativo que o grau de condensação pode ter sido menos para essa amostra, obtendo-se assim um grau de condensação de aproximadamente 2:1 (TEOS:MTMS).

Alguns autores atribuem que o grau de condensação é dependente do tipo de catálise utilizada. O uso de catálise ácida conduz a aproximadamente $70 \%$ de condensação, e a básica à $90 \%$, porém outros
Tabela 2. Resultados da análise elementar de CNH para as amostras; experimentais (E) e calculados $(\mathrm{T})$.

\begin{tabular}{ccccccc}
\hline \multirow{2}{*}{ amostras } & \multicolumn{2}{c}{$\mathrm{C}(\%)$} & \multicolumn{2}{c}{$\mathrm{H}(\%)$} & \multicolumn{2}{c}{$\mathrm{N}(\%)$} \\
\cline { 2 - 7 } & $\mathrm{T}$ & $\mathrm{E}$ & $\mathrm{T}$ & $\mathrm{E}$ & $\mathrm{T}$ & $\mathrm{E}$ \\
\hline $\mathrm{S} 1$ & 0 & 0 & 0 & 0 & 0 & 0 \\
$\mathrm{~S} 2$ & 0 & 0 & 0 & 0 & 0 & 0 \\
S3 & 9,53 & 10,94 & 2,36 & 1,44 & 0 & 0 \\
S4 & 9,53 & 6,70 & 2,36 & 1,98 & 0 & 0 \\
S5 & 22,5 & 13,71 & 5,00 & 3,75 & 8,70 & 6,16 \\
S6 & 22,5 & 15,21 & 5,00 & 4,08 & 8,70 & 6,24 \\
S7 & 18,90 & 11,62 & 3,15 & 2,14 & 0 & 0 \\
S8 & 18,90 & 16,93 & 3,15 & 2,99 & 0 & 0 \\
\hline
\end{tabular}

Obs: Os cálculos foram realizados levando em consideração a formação da sílica $1: 1$.

fatores podem influenciar na condensação, tais como: solvente, concentração dos reagentes, tipo de substituinte no percursor silano, etc. ${ }^{18}$. No caso das amostras $\mathbf{S 3}$ e $\mathbf{S 4}$ observamos que a catálise ácida favoreceu uma maior porcentagem de condensação para o silano MTMS.

Os mecanismos propostos na literatura ${ }^{19}$ para a catalise ácida e básica são representados na Figura 3.

As amostras S5, S6, S7 e S8 apresentaram valores inferiores aos esperados para $\mathrm{C}, \mathrm{H}$ e $\mathrm{N}$, indicando que o grau de condensação foi influenciado pelo substituinte. Porém nestes casos as amostras catalisadas pela base obtiveram um maior grau de hidrólise.

Através da microscopia eletrônica de varredura notamos que as partículas de sílica formadas possuem formas irregulares. O tamanho varia muito, porém para a amostra $\mathbf{S 1}$, enquanto que para a $\mathbf{S 2}$ as partículas foram menores.

Já nas amostras S3 e S4 também foram observadas formas irregulares e tamanhos diferentes de partículas, sendo as maiores partículas obtidas a partir da catálise básica.

A partir da amostra $\mathbf{S 5}$ começam a surgir partículas com forma esférica. A Figura 4 mostra as microscopia eletrônica de varredura para as amostras $\mathbf{S 7}$ e $\mathbf{S 8}$.

Dois fatos chamam a atenção, o tamanho da esfera e a sua superfície. Na amostra $\mathbf{S 7}$ observamos partículas com diâmetros variados e uma superfície aparentemente lisa. Já na amostra $\mathbf{S 8}$ observamos uma maior homogeneidade no tamanho das partículas, porém em relação à sua superfície notamos uma rugosidade nas partículas. A rugosidade das partículas pode indicar que os grupamentos orgânicos pertencentes ao silano cloropropil estão voltados para o exterior das partículas, o que seria de grande interesse para esse trabalho. Isto porque nessas partículas de aproximadamente $1 \mu \mathrm{m}$ de diâmetro podem ser utilizados para suporte de materiais luminescentes. O tamanho e superfície também foram influenciadas pela tipo de catalisador utilizado.

A presença das moléculas orgânicas nas partículas de sílicas, foram qualitativamente confirmada através da espectroscopia de absorção no infravermelho (IV).

Os espectros de IV apresentaram os modos vibracionais da sílica em $460 \mathrm{~cm}^{-1}$ correspondente a $\delta(\mathrm{Si}-\mathrm{O}-\mathrm{Si}), 785 \mathrm{~cm}^{-1} v_{\mathrm{s}}(\mathrm{Si}-\mathrm{O}-\mathrm{Si})$, $940 \mathrm{~cm}^{-1} v(\mathrm{Si}-\mathrm{OH})$ e $1085 \mathrm{~cm}^{-1} v_{\text {as }}(\mathrm{Si}-\mathrm{O}-\mathrm{Si})^{20}$.

Vibrações originárias da absorção de moléculas de água na região de $3500 \mathrm{~cm}^{-1}$ e $1635 \mathrm{~cm}^{-1}{ }^{21}$, também foram observadas, indicando a presença de grupos -OH na superfície, isto mostra que o grau de condensação não foi total. A banda em $3750 \mathrm{~cm}^{-1}$ confirma a presença de $\mathrm{OH}$ livre ${ }^{20}$.

Em geral observamos uma diminuição da banda ao redor de 950 $\mathrm{cm}^{-1}$, esta absorção é atribuída a grupos -OH ligado ao Si. Esta diminuição está relacionada à presença dos grupos orgânicos na superfície da sílica. 
MECANISMOS:

\section{CATÁLISE ÁCIDA}

Hidrolise

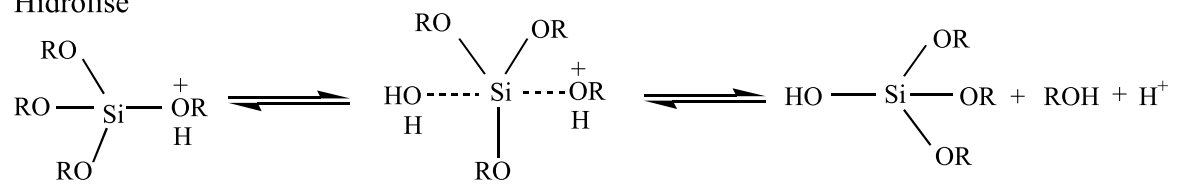

\section{Condensação}<smiles></smiles>

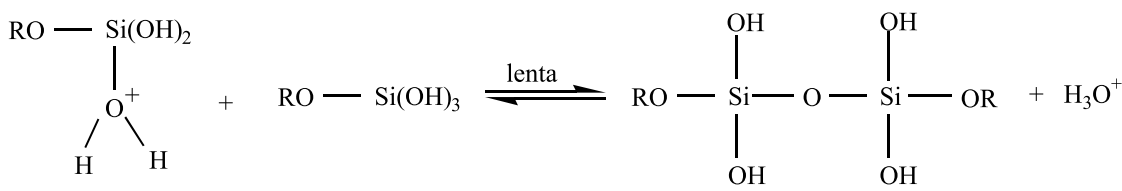

\section{CATÁLISE BÁSICA}

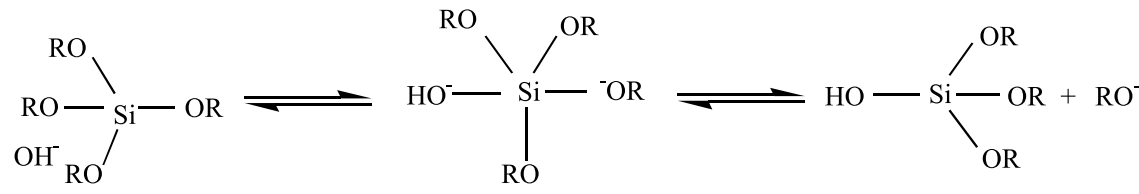

\section{Condensação}

$$
\begin{aligned}
& \mathrm{RO}-\mathrm{Si}(\mathrm{OH})_{3}+\mathrm{OH}^{-} \stackrel{\text { rapida }}{=} \mathrm{RO}-\mathrm{Si}(\mathrm{OH})_{2} \mathrm{O}^{-}+\mathrm{H}_{2} \mathrm{O} \\
& \mathrm{RO}-\mathrm{Si}(\mathrm{OH})_{3}+\mathrm{RO}-\mathrm{Si}(\mathrm{OH})_{2} \mathrm{O}^{-} \stackrel{\text { lenta }}{=} \mathrm{RO}-\mathrm{Si}(\mathrm{OH})_{2}-\mathrm{O}-\mathrm{Si}(\mathrm{OH})_{2} \mathrm{OR}+\mathrm{OH}^{-}
\end{aligned}
$$

Figura 3. Mecanismos para as reações de catalise ácida e básica ${ }^{19}$.
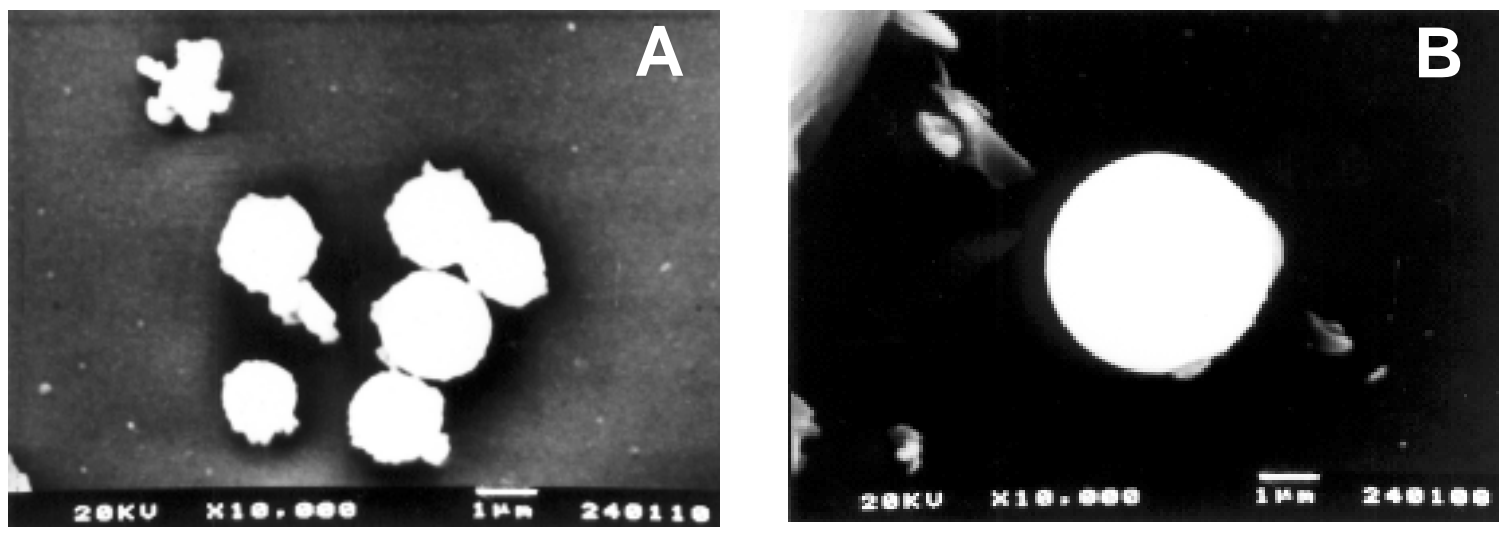

Figura 4. Microscopia eletrônica (MEV) das amostras, a) S8 e b) S7. 
Nas amostras S3 e S4 vibrações -CH (2973 e $\left.1282 \mathrm{~cm}^{-1}\right)$ indicam a presença dos grupos $-\mathrm{CH}$ originários do metiltrimetoxissilano (MTMS). O aparecimento de uma larga banda na região de $3400 \mathrm{~cm}^{-}$ ${ }^{1}$ para a amostra S4 indica uma presença maior de moléculas de água na amostra. Isto pode ser visto através da análise elementar, onde a quantidade de carbono encontrada foi menor que a encontrada para amostra S3. Sugerindo um grau de condensação para esta amostra S4 menor que a da S3.

Os espectros das amostras S5 e S6 (TEOS-APTS) mostraram-se muito semelhantes, apresentando modos vibracionais característicos do grupamento orgânico (amino propil). Em 2938 e $2881 \mathrm{~cm}^{-1}$ aparecem as vibrações assimétricas e simétricas $\mathrm{CH}$ e deformação $\mathrm{CH}$ em $1480 \mathrm{~cm}^{-1}$ e também vibrações referentes ao grupo $\mathrm{NH}_{2}$ em $3300,3200,1560$ e $1490 \mathrm{~cm}^{-1} 21,22$.

As amostras S7 e S8 apresentaram vibrações características de grupos $\mathrm{CH}_{2}$ em 2950 e $1450 \mathrm{~cm}^{-121}$.

Na Tabela 3 apresentados as posições dos picos no espectro de ressonância magnética nuclear do silício nas diferentes amostras.

Tabela 3. Posição dos picos no RMN ${ }^{29} \mathrm{Si}$, referente aos diferentes tipos de silício existente nas amostras.

\begin{tabular}{cccccccc}
\hline \multirow{2}{*}{ Amostras } & \multicolumn{7}{c}{ Deslocamento químico / ppm } \\
\cline { 2 - 8 } & $\mathrm{T}_{1}$ & $\mathrm{~T}_{2}$ & $\mathrm{~T}_{3}$ & $\mathrm{Q}_{1}$ & $\mathrm{Q}_{2}$ & $\mathrm{Q}_{3}$ & $\mathrm{Q}_{4}$ \\
\hline S1 & - & - & - & - & -93 & -103 & -112 \\
S2 & - & - & - & - & - & -103 & -113 \\
S3 & - & -58 & -66 & - & - & -102 & -111 \\
S4 & - & -57 & -67 & - & - & -103 & -112 \\
S5 & - & -60 & -69 & - & - & -100 & -109 \\
S6 & - & -49 & -67 & - & - & -102 & -109 \\
S7 & - & -58 & -66 & - & - & -101 & -109 \\
S8 & - & -57 & -67 & - & - & -102 & -110 \\
\hline
\end{tabular}

Nas amostras S1 e S2 notamos picos referentes somente as espécies de silício, como mostra a Figura $5^{24}$.

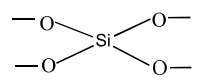

$\mathrm{Q}_{4}$<smiles>CO[Si](O)(O)O[Si](O)(OC)OC</smiles>

$\mathrm{Q}_{3}$

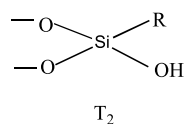

$\mathrm{T}_{2}$

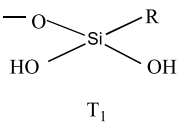

$\mathrm{R}=-\mathrm{CH}_{3} ;-\left(\mathrm{CH}_{2}\right)_{3} \mathrm{Cl} ;-\left(\mathrm{CH}_{2}\right)_{3} \mathrm{NH}_{2}$

onde: $Q$ indica átom os de silício ligados a átomos de oxigênio. Os índices o número de átomos de oxigênio interligando dois átomos de silício.

$\mathrm{T}$ indica átomos de silício ligados a átomos de carbono.

Figura 5. Espécies de silício presente na sílica.

Nos espectros de RMN Si apresenta um pico ao redor de 70 ppm, o qual foi atribuído ao rotor utilizado na obtenção dos espectros. O espectro da amostra $\mathrm{S} 1$ apresenta o pico referente ao silício $\mathrm{Q}_{2}$, o que não acontece com a S2. As abundâncias das espécies para a $\mathrm{S} 1$ aparecem na proporção 1:4:4: respectivamente a $\mathrm{Q}_{2}, \mathrm{Q}_{3}, \mathrm{Q}_{4}$. Para as $\mathrm{S} 21: 2, \mathrm{Q}_{3}: \mathrm{Q}_{4}$. Observando estes valores notamos a dependência do grau de hidrólise e condensação com relação ao tipo de catalisador utilizado. A Figura 6 a e b apresentam os espectros de RMN Si para as amostras S1(a) e S2(b).

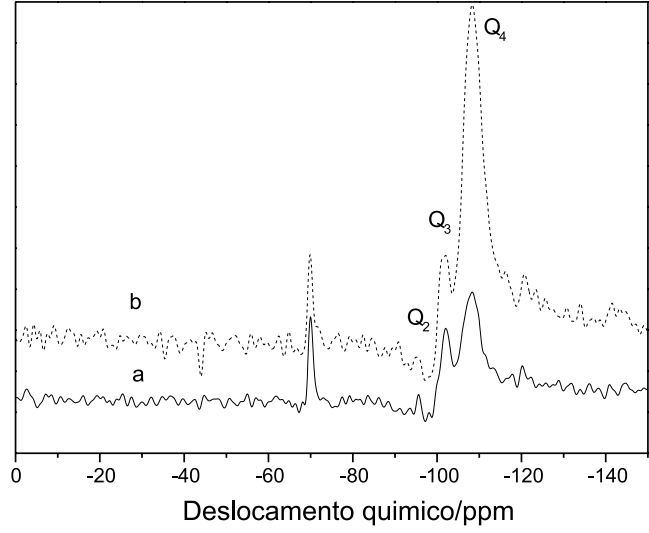

Figura 6. Espectros de RMN Si nas amostras, a) S1 e b) S2.

A observação do sinais relativos a átomo de silício $\mathrm{Q}_{1}, \mathrm{Q}_{2}, \mathrm{Q}_{3} \mathrm{e}$ $\mathrm{Q}_{4}$ é possível devido a deslocamentos químicos da ordem de $10 \mathrm{ppm}$ $\left(\mathrm{Q}_{4} \sim-110 \mathrm{ppm}, \mathrm{Q}_{3} \sim-100 \mathrm{ppm}\right.$, etc). A mudança de uma ligação Si$\mathrm{O}$ para $\mathrm{Si}-\mathrm{C}$, transforma silício $\mathrm{Q}$ em silício T causando uma mudança de aproximadamente 45 ppm e uma separação entre eles também de $10 \mathrm{ppm}\left(\mathrm{T}_{3} \sim-65 \mathrm{ppm}, \mathrm{T}_{2} \sim-55 \mathrm{ppm} \text {, etc }\right)^{25}$.

A Tabela 4 apresenta as proporções entre espécies de silício nas amostras, calculadas através da integração dos picos.

Tabela 4. Proporção entre os tipos de silício nas amostras obtidos.

\begin{tabular}{cccccccc}
\hline Amostras & $\mathrm{T}_{1}$ & $\mathrm{~T}_{2}$ & $\mathrm{~T}_{3}$ & $\mathrm{Q}_{1}$ & $\mathrm{Q}_{2}$ & $\mathrm{Q}_{3}$ & $\mathrm{Q}_{4}$ \\
\hline S1 & - & - & - & - & 1 & 4 & 4 \\
S2 & - & - & - & - & - & 1 & 2 \\
S3 & - & 1 & 3 & - & - & 2 & 3 \\
S4 & - & 1 & 3 & - & - & 2 & 3 \\
S5 & - & 2 & 3 & - & - & 2 & 1 \\
S6 & - & 2 & 1 & - & - & 3 & 2 \\
S7 & - & 1 & 3 & - & - & 3 & 2 \\
S8 & - & 1 & 3 & - & - & 2 & 3 \\
\hline
\end{tabular}

Não foram observadas diferenças significativas para as amostras S3 e S4, preparadas a partir do TEOS e MTMS, diferindo apenas no catalisador $\left(\mathrm{HCl}\right.$ e $\left.\mathrm{NH}_{4} \mathrm{OH}\right)$. Neste caso portanto o tipo de catálise não influenciou na condensação dos alcóxidos. $\mathrm{O}$ aparecimento dos picos $\mathrm{T}_{2}$ e $\mathrm{T}_{3}$ confirma a existência de átomos de carbono ligados ao silício, fato que já havia sido observado através dos espectros de infravermelho. Na Figura 7 a e b são mostrados os espectros de RMN Si para as amostras S3 e S4.

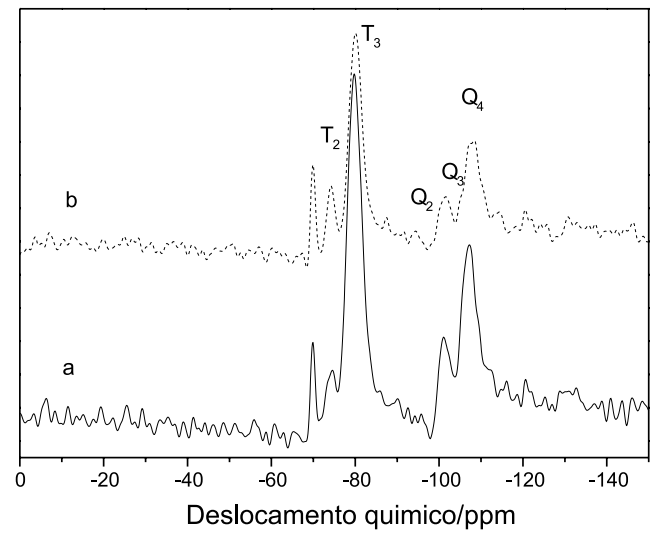

Figura 7. Espectros de RMN Si das amostras, a) S3 e b) S4. 
As amostras S5 e S6, contendo o aminopropil como modificador da superfície, apresentaram uma inversão na porcentagem de $\mathrm{Q}_{3} \mathrm{e}$ $\mathrm{Q}_{4}$, isto é, na amostra catalisada por ácido (S5) ocorreu um favorecimento na formação de sítios $\mathrm{Q}_{3}$ de silício. $\mathrm{O}$ mesmo foi observado para as amostras S7 e S8.

Os espectros de RMN de ${ }^{13} \mathrm{C}$ para as amostras S3 e S4 apresentaram apenas um sinal em $-2,9$ ppm. Segundo Kallury ${ }^{26}$ este sinal é atribuído a um grupo metil $\left(-\mathrm{CH}_{3}\right)$ ligado ao silício. Para as amostras S5, S6, S7 e S8 observou-se três distintos sinais em 12,1; 23,4 e 43,9 ppm, atribuídos aos três carbonos do grupo propil com origem nos precursores cloropropil e aminopropil. A integração das áreas sob os picos apresentaram uma razão de 1:1:1, indicando a mesma quantidade de átomos carbono. Temos carbono ligado ao silício $\left(-\mathrm{CH}_{2}-\mathrm{Si}\right)$; carbono ligado a outros dois carbono $\left(\mathrm{C}-\mathrm{CH}_{2}-\mathrm{C}\right)$ e finalmente carbono ligado ao cloro ou grupo amino $\left(\mathrm{Cl}-\mathrm{CH}_{2}-\mathrm{Ou} \mathrm{NH}_{2}-\mathrm{CH}_{2}-\right)$.

\section{CONCLUSÃO}

- Os tipos de catalisadores utilizados $\left(\mathrm{HCl}\right.$ e $\left.\mathrm{NH}_{4} \mathrm{OH}\right)$ influenciaram no tipo e na proporção de silício formado a partir da hidrólise do tetraetilortossilicato;

- A utilização de $\mathrm{HCl}$ favoreceu principalmente a formação de sítios $\mathrm{Q}_{3}$, enquanto que a utilização de $\mathrm{NH}_{4} \mathrm{OH}$ favoreceu a formação $\mathrm{Q}_{4}$.

- As técnicas de absorção no infravermelho e análise elementar indicaram a formação da sílica funcionalizada pelo método solgel. A presença dos grupos orgânicos no polímero inorgânico também foi confirmada dos resultados de ressonância magnética nuclear das sílicas no estado sólido (RMN $\left.{ }^{29} \mathrm{Si} \mathrm{e}^{13} \mathrm{C}\right)$.

\section{AGRADECIMENTOS}

Os autores agradecem à Fundação de Amparo à Pesquisa do Estado de São Paulo (FAPESP) pelo suporte financeiro e bolsa concedida e a Silvia H. S. Benedicto do IQ-UNESP pelos espectros de RMN.

\section{REFERÊNCIAS}

1. Farias, R. F. de; Airoldi, C.; Quim. Nova 2000, 23, 88.

2. Mitchell, S. A.; Chem. Ind. 1966, 4, 924.

3. Armistead, C. G.; Tyler, A J.; Hambleton, F. H.; Mitchell, S. A.; Hockey, J. A; J. Phys. Chem. 1969, 73, 3947.

4. Gushikem, Y.; Moreira, J. C.; J. Colloid Interface Sci. 1984, 107, 70.

5. Iamamoto, Y.; Prado, C. M. C. ; Sacco, H. C.; Ciuffi, K. J.; Assis, M. D.; Maestrin, A. P. J.; Melo, A. J. B.; Baffa, O.; Nascimento, O R.; J. Mol. Catal. 1997, 117, 259.

6. Jin, T.; Inoue, S.; Machida, K.; Adachi, G.; J. Alloys Comp. 1998, 265, 234.

7. Nassar, E. J.; Serra, O. A. ; Quim. Nova 2000, 23, 16.

8. Airoldi, C.; Farias, R. F. de; Quim. Nova 2000, 23, 496.

9. Wells, S.D.; Koopal, L.K.; de Keizer, A.; Colloid Surf. A. 2000, 166, 171.

10. Markowitz, M. A.; Schoen, P. E.; Just, P.; Gaber, B. P.; Colloid Surf. A Physicochem. Eng. Aspects 1999, 150, 85.

11. Vrancken, K. C.; Van Der Voort, P.; Possemiers, K.; Vansant, E. F, J. Colloid Interface Sci. 1995, 174, 86.

12. Nassar, E. J.; Neri, C. R.; Calefi, P. S.; Serra, O. A.; J. Non-Cryst. Solids 1999, 147, 124.

13. Serra, O. A.; Nassar, E. J.; Zapparolli, G.; Rosa, I. L. V.; J Alloys Comp. 1994, 207/208, 454.

14. Nassar, E. J.; Rosa, I. L. V.; Serra, O. A.; J. Alloys Comp. 1997, 250, 380.

15. Serra, O. A.; Nassar, E. J.; Rosa, I. L. V.; J. Lumin. 1997, 72-74, 263.

16. Serra, O. A., Nassar, E. J., Zapparolli, G.; Rosa, I. L .V.; J. Alloy Comp. 1995, 225,63.

17. Green, W. H.; Le, K. P.; Grey, J.; Au, T. T.; Sailor, M. J.; Science 1997, 276, 1826; - Carlos, L. D.; Sá Ferreira, R. A.; Zéa Bermudez, V.; Ribeiro, S. J. L.; Advanced Functional Materials 2001,11, 111.

18. Corriu, R. P. J.; Leclerq, D.; Angew. Chem. Int. Ed. Engl. 1996, 35 , 1421.

19. Buckley, A. M.; Greenblatt, M.; J. Chem. Educ. 1994, 71, 599.

20. Perry, C. C.; Li, X.; Waters, D. N.; Spectrochim. Acta 1991, 47A, 1487.

21. Nakamoto, K., In Infrared and Raman of Inorganic and Coordination Compounds, 4rd ed. Willey, New York, 1986.

22. Bekiari, V.; Lianos, P.; Langmuir 1998, 14, 3459.

23. Chiang, C. H.; Ishida, H.; Koenig, S. L.; J. Colloid Interface Sci. 1980, $74,396$.

24. Badley, R. D.; Ford, W. T.; McEnroe, F. J.; Assink, R. A.; Langmuir 1990, 6, 792 .

25. Blaaderen, A. van; Vrij, A.; J. Colloid Interface Sci. 1993, 156, 1.

26. Kalluray, K. M. R.; Macdonald, P. M.; Thompson, M.; Langmuir 1994, $10,492$. 